\title{
Adiabatic compression of parallel-plate metal waveguides for sensitivity enhancement of waveguide $\mathrm{THz}$ time-domain spectroscopy
}

\author{
Jiangquan Zhang and D. Grischkowsky ${ }^{\mathrm{a})}$ \\ School of Electrical and Computer Engineering, Oklahoma State University, Stillwater, Oklahoma 74078
}

(Received 5 November 2004; accepted 14 December 2004; published online 2 February 2005)

\begin{abstract}
We demonstrate the efficient coupling of $\mathrm{THz}$ pulses into and out of an adiabatically compressed parallel-plate metal waveguide. The plate separation of the $63.5-\mathrm{mm}$ long, compressed waveguide was fixed at $103 \mu \mathrm{m}$ at the entrance and exit ends for efficient coupling, but could be reduced in the middle. The experimental results show strong transmission of the $\mathrm{THz}$ signal through the compressed waveguide for a minimum midpoint separation of only $8 \mu \mathrm{m}$. This technique can be used to achieve efficient coupling together with higher sensitivity for waveguide THz-TDS. (C) 2005 American Institute of Physics. [DOI: 10.1063/1.1863439]
\end{abstract}

THz waveguides have recently been developed with low loss and single-mode propagation. ${ }^{1-5}$ Among the several types of waveguides studied, the parallel-plate metal waveguides are of special interest due to their associated low-loss and low-group velocity dispersion in the transverseelectromagnetic (TEM)-mode propagation. ${ }^{3,4}$ With these developments, planar $\mathrm{THz}$ quasioptics and interconnects have also been realized. ${ }^{5,6} \mathrm{THz}$ waveguides can also be used for spectroscopy purposes. ${ }^{1}$ Waveguide THz-TDS, using the parallel-plate metal waveguide, has demonstrated exceptional sensitivity by probing nanometer water layers. ${ }^{7}$ The sensitivity of waveguide THz-TDS is inversely proportional to the separation between the two plates forming the waveguide, ${ }^{1,7}$ so it is desirable to minimize the plate separation.

Currently, quasioptic techniques incorporating cylindrical silicon lenses are used to couple the $\mathrm{THz}$ beam into and out of the waveguide. This coupling is strongly dependent on the plate separation of the waveguide; minimized plate separation will reduce the coupling. For example, if the same cylindrical lens pair is used, the amplitude coupled into a parallel-plate waveguide with $10-\mu \mathrm{m}$ plate separation would be approximately $10 \%$ of that with $100-\mu \mathrm{m}$ plate separation; the same logic holds for the output, giving a throughput of $1 \%$ for the waveguide with $10-\mu \mathrm{m}$ separation compared with the throughput for $100-\mu \mathrm{m}$ separation. This strongly reduced coupling will significantly reduce the signal-to-noise ratio in the spectroscopy, thereby defeating the gain in sensitivity achieved by reducing the plate separation.

Tapered transmission lines, ${ }^{8,9}$ and tapered waveguides ${ }^{10,11}$ have long been used in the microwave region to match unequal waveguide impedances and to connect guiding devices with different cross sections. When the taper is properly designed, the reflection loss and mode conversion can be neglected for the guided electromagnetic wave. In this letter we demonstrate the coupling of $\mathrm{THz}$ pulses into and out of a compressed parallel-plate metal waveguide with an adiabatically varying plate separation, thereby enabling the efficient coupling of $\mathrm{THz}$ waves to a metal waveguide with a narrow plate separation. The standard confocal THz-TDS system $^{12}$ and the compressed waveguide structure used for this experiment are shown in Fig. 1. The metal waveguide consists of two identical copper plates, with dimensions of $63.5 \mathrm{~mm}$ (length) $\times 27.9 \mathrm{~mm}$ (width) $\times 9.5 \mathrm{~mm}$ (thickness). The copper plates were hand-polished to a mirror finish with submicron flatness. The cylindrical silicon lenses used in this experiment, with dimensions of $15 \mathrm{~mm}$ (height) $\times 6.56 \mathrm{~mm}$ (thickness) $\times 5 \mathrm{~mm}$ (radius), are attached $50 \mu \mathrm{m}$ from the entrance and exit ends of the waveguide. The incoming THz beam is horizontally $x$-polarized, perpendicular to the waveguide opening and the cylindrical axes of the lenses. The entire waveguide structure is placed at the confocal $\mathrm{THz}$ beam waist midway between the two off-axis paraboloidal mirrors. ${ }^{6}$ The THz-TDS system and the waveguide structure are placed in an air-tight box purged with dry air to mitigate the absorption lines of water vapor.

The two copper plates are separated at the four corners by brass spacers with dimensions of $3 \mathrm{~mm}$ (length, along the waveguide openings) $\times 2 \mathrm{~mm}$ (width) $\times 103 \mu \mathrm{m}$ (thickness), determining the $103-\mu \mathrm{m}$ plate separation at the entrance and exit ends of the waveguide. A stainless steel compressor is applied at the midpoint of the waveguide to vary the mid-

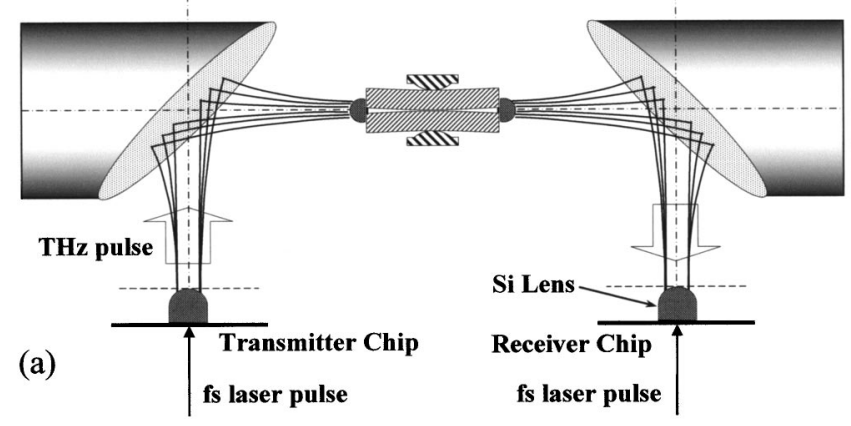

(b)

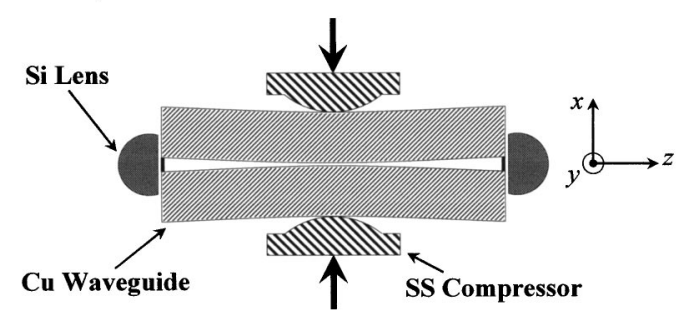

FIG. 1. (a) Experimental setup and (b) the adiabatically compressed waveguide structure.
a)Phone: 405-744-6622; Fax: 405-744-9198; electronic mail: grischd@ceat.okstate.edu 

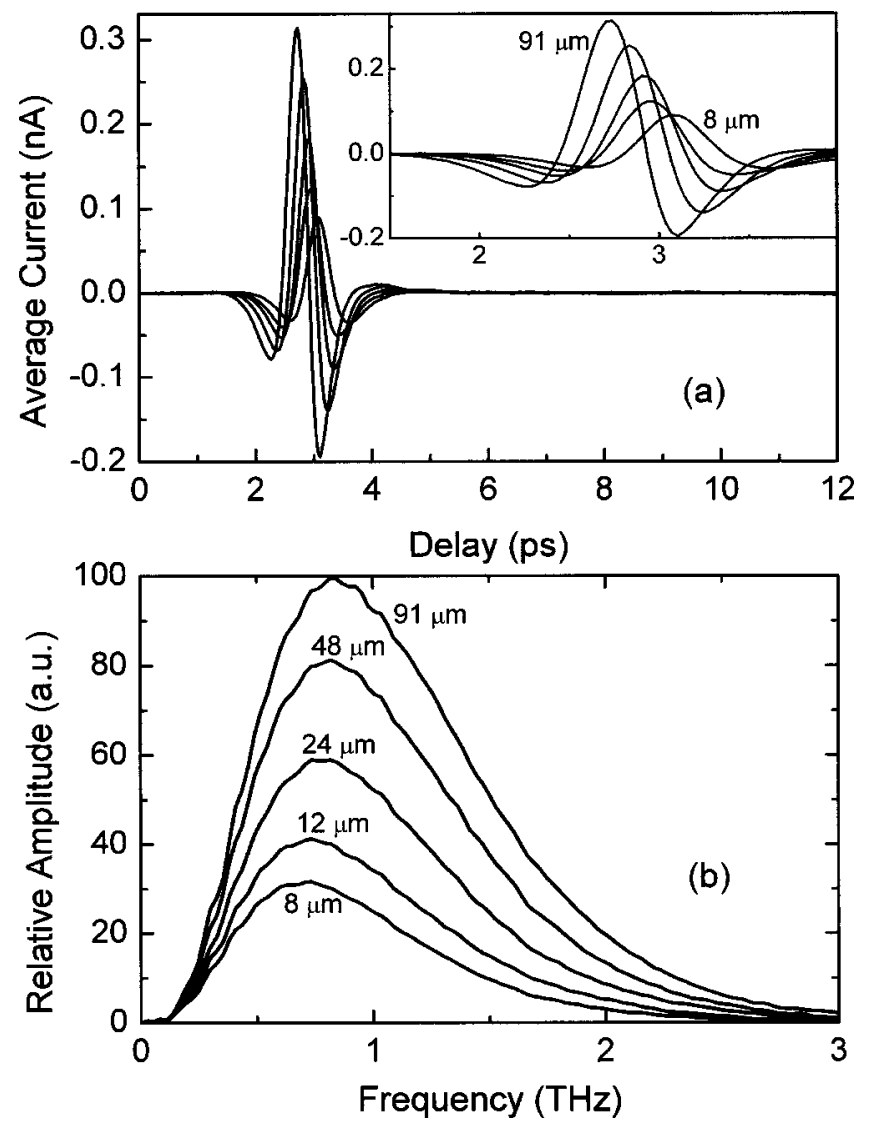

FIG. 2. (a) Output $\mathrm{THz}$ pulses from the compressed waveguide with midpoint separations of 91 (reference), 48, 24, 12, and $8 \mu \mathrm{m}$. The inset is the zoom-in view of the pulses. (b) The respective spectra.

point plate separation in the range from 0 to $103 \mu \mathrm{m}$. The compressor is composed of two identical hypercylindrical stainless steel plates with a radius of curvature of $38 \mathrm{~mm}$. For reference, the waveguide is at minimal compression with the measured midpoint plate separation of $91 \mu \mathrm{m}$. The attached cylindrical lenses are carefully aligned to optimize the $\mathrm{THz}$ signal passed through the waveguide. After a reference scan is taken, the waveguide is compressed to change the midpoint plate separation. This compression is examined under a microscope to make sure that the separation is the same for both the top and bottom edges, and therefore is uniform along the mid-line between the plates.

Figure 2(a) shows the $\mathrm{THz}$ reference pulse transmitted through the waveguide with $91-\mu \mathrm{m}$ midpoint separation and those taken with midpoint separations of $48,24,12$, and $8 \mu \mathrm{m}$, with each separation having an uncertainty of $\pm 3 \mu \mathrm{m}$. The respective spectra are presented in Fig. 2(b). From Fig. 2 , it is seen that the transmitted $\mathrm{THz}$ pulse for the compressed waveguide with the minimal 8 - $\mu$ m midpoint separation is only reduced to $30 \%$ of that for the reference pulse with $91-\mu \mathrm{m}$ midpoint separation. This result is fully explained by the increased absorption of the compressed waveguide; the coupling remains the same for the two cases.

The inset of Fig. 2(a) is the zoom-in view of the transmitted $\mathrm{THz}$ pulses, showing that as the midpoint plate separation is reduced, the attenuated $\mathrm{THz}$ pulse moves to later delays; the delay for the minimal $8-\mu \mathrm{m}$ separation is $0.35 \mathrm{ps}$ compared with the reference pulse. It is also seen that, the full-width at half-maximum of the pulse broadened slightly with compression, from $0.30 \mathrm{ps}$ for the reference $91-\mu \mathrm{m}$ pulse to 0.33 ps for the 8 - $\mu \mathrm{m}$ output pulse. In our case, the THz pulse propagates in the TEM mode of the parallel-plate waveguide. ${ }^{3}$ With perfect conductor plates, the TEM mode propagation would be free of group velocity dispersion and power absorption; hence the pulse delay and broadening should not be observed for the compressed waveguide. However, the finite conductivity of the metal plates causes the slight group velocity dispersion, the increase of the propagation constant, and the frequency-dependent absorption, resulting in the observed time delay, pulse amplitude reduction, and pulse broadening.

For the TEM mode propagation of a parallel-plate waveguide, the amplitude absorption coefficient caused by the finite conductivity of the metal is determined by $\alpha(\omega)$ $=R_{S} / \eta_{0} b,{ }^{13}$ where $\omega$ is the angular frequency, $\eta_{0}=377 \Omega$ is the intrinsic impedance of free space, $\mathrm{b}$ is the plate separation of the waveguide, $R_{s}=\sqrt{\omega \mu_{0} / 2 \sigma}, \mu_{0}$ is the free-space permeability, and $\sigma$ is the conductivity. We use $\sigma=5.8$ $\times 10^{7} \Omega^{-1} \mathrm{~m}^{-1}$ for copper. In general for a waveguide with cross-sectional change, the attenuation is caused by both the absorption due to the finite conductivity and the reflection due to the cross-sectional change. However, if the crosssectional change is made adiabatically (slowly) over a length of many wavelengths, the reflection is negligible. ${ }^{9,10}$ In our case, the cross-sectional change is made in a length of $30 \mathrm{~mm}, 100$ times the wavelength at $1 \mathrm{THz}$, thus the reflection can be neglected. The absorption coefficient is therefore only a function of plate separation, i.e., $\alpha(\omega, z)$ $=\alpha_{0}(\omega) b_{0} / b(z)$, where $b_{0}$ is the plate separation at the ends, and $\alpha_{0}(\omega)=R_{s} / \eta_{0} b_{0}$. For a straight waveguide with length $L$ and uniform plate separation $b_{0}$, the total amplitude attenuation due to the finite conductivity of the metal plates would be $A_{0}(\omega)=\exp \left[-\alpha_{0}(\omega) L\right]$. For the compressed waveguide, the total amplitude attenuation can be calculated correspondingly as

$$
A_{c}(\omega)=\exp \left[-\int_{-L / 2}^{L / 2} \alpha(\omega, z) d z\right]=\exp \left[-\alpha_{0}(\omega) L\left(b_{0} / b_{E}\right)\right],
$$

where

$$
b_{0} / b_{E}=\left(b_{0} / L\right) \int_{-L / 2}^{L / 2}[1 / b(z)] d z .
$$

It is quite important to notice that the effect of the adiabatic waveguide compression as displayed in Eqs. (1) and (2) does not change the frequency dependence of $\alpha_{0}(\omega)$ but only changes the absorption strength by the compression factor $\left(b_{0} / b_{E}\right)$. This feature can enable very precise determinations of the frequency dependence of $\alpha_{0}(\omega)$ using waveguide THzTDS with adiabatic compression.

For a simply supported elastic beam of length $L$, when pressed in the middle, the cross section deflection curve takes the form of $x \propto z^{\prime}\left(3 L^{2}-4 z^{\prime 2}\right),{ }^{14}$ where $z^{\prime}=L / 2-|z|$ and $-L / 2 \leqslant z \leqslant L / 2$. In our case, we can assume that the ends of copper plates move freely when compressed, so that the compressed waveguide plates can be treated as simply supported beams. The plate separation of the compressed waveguide can therefore be written as $b(z)=b_{0}-p z^{\prime}\left(3 L^{2}-4 z^{\prime 2}\right)$, where $p=\left(b_{0}-b_{m}\right) / L^{3}$, and $b_{m}$ is the midpoint plate separation. Note that $z=0$ at the midpoint of the waveguide. To test this prediction, we measured the plate separation across the 


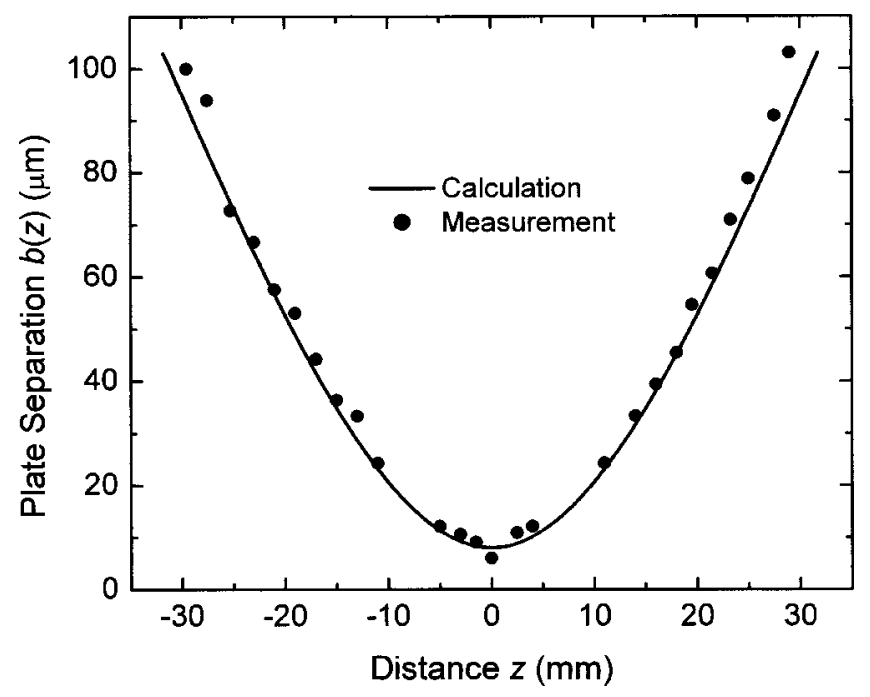

FIG. 3. Comparison of the measured (dots) and calculated (solid line) plate separations for the compressed waveguide with $8-\mu \mathrm{m}$ midpoint plate separation.

compressed waveguide for an 8 - $\mu \mathrm{m}$ midpoint separation. The measured results and the calculated results based on the simply supported beam assumption are compared in Fig. 3. It can be seen that, the measurement and the calculation agree reasonably well; the difference between measurement and calculation is less than $5 \mu \mathrm{m}$ for most of the data points.

With the above analysis we can now compare the amplitude absorption for the reference guide with the compressed guide. Since the cylindrical lenses and the plate separation at the ends are fixed, the coupling between the cylindrical lenses and the waveguide is considered to be the same for the reference guide and the compressed guides. Thus, the pulse and spectrum changes are solely caused by the different absorption of the compressed guides. We can therefore compare the scan from the reference guide with individual scans from the compressed guides in terms of the amplitude spectral ratio, which gives the relative absorption information. Figure 4 shows the amplitude spectral ratios between scans from the compressed waveguides and the reference guide. Using Eq. (2), the compression factors $\left(b_{0} / b_{E}\right)$ are calculated to be $1.08,1.59,2.38,3.50$, and 4.37 for the midpoint separations of $91,48,24,12$, and $8 \mu \mathrm{m}$, respectively. The calculated spectral ratios (solid lines) match the experimental values (dotted lines) reasonably well for all the cases.

The experimental results and the simple analysis demonstrate and explain the efficient coupling of $\mathrm{THz}$ pulses into and out of an adiabatically compressed parallel-plate metal waveguide. Using this technique, it is possible to achieve

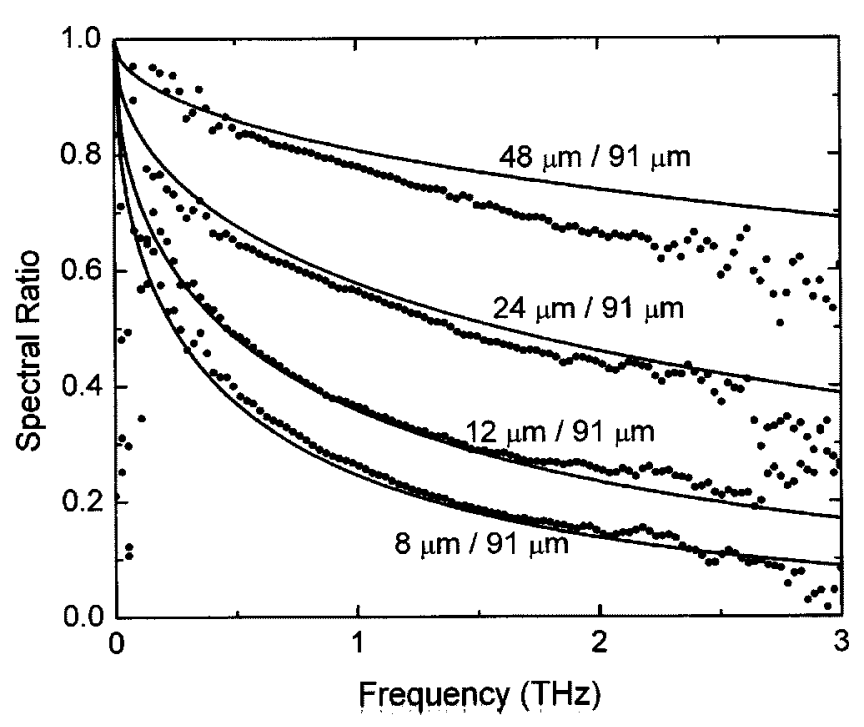

FIG. 4. Spectral ratios between the transmitted THz pulses from compressed waveguide and that from the reference guide. Experiment (dots); theory (solid lines).

both high sensitivity and good coupling to significantly improve the sensitivity of waveguide THz-TDS. Because adiabatic compression does not change the frequency dependence of the absorption coefficient, this technique can determine this dependence with increased sensitivity and accuracy.

The authors acknowledge Darpan Pradhan, Sarika Pokharel, and Kiran Joshi for their invaluable experimental assistance. This work was partially supported by the National Science Foundation, the U.S. Army Research Office, and the Department of Energy.

${ }^{1}$ G. Gallot, S. P. Jamison, R. W. McGowan, and D. Grischkowsky, J. Opt. Soc. Am. B 17, 851 (2000).

${ }^{2}$ R. Mendis and D. Grischkowsky, J. Appl. Phys. 88, 4449 (2000).

${ }^{3}$ R. Mendis and D. Grischkowsky, Opt. Lett. 26, 846 (2001).

${ }^{4}$ R. Mendis and D. Grischkowsky, IEEE Microw. Wirel. Compon. Lett. 11, 444 (2001)

${ }^{5}$ S. Coleman and D. Grischkowsky, Appl. Phys. Lett. 83, 3656 (2003).

${ }^{6}$ J. Dai, S. Coleman, and D. Grischkowsky, Appl. Phys. Lett. 85, 884 (2004).

${ }^{7}$ J. Zhang and D. Grischkowsky, Opt. Lett. 29, 1617 (2004).

${ }^{8}$ R. W. Klopfenstein, Proc. IRE 44, 31 (1956).

${ }^{9}$ R. E. Collin, Proc. IRE 44, 539 (1956).

${ }^{10}$ K. Matsumaru, IRE Trans. Microwave Theory Tech. 6, 143 (1958).

${ }^{11}$ H.-G. Unger, Bell Syst. Tech. J. 37, 899 (1958).

${ }^{12}$ D. Grischkowsky, S. Keiding, M. van Exter, and Ch. Fattinger, J. Opt. Soc. Am. B 7, 2006 (1990).

${ }^{13}$ C. A. Balanis, Advanced Engineering Electromagnetics (Wiley, New York, 1989).

${ }^{14}$ R. C. Hibbeler, Mechanics of Materials (Macmillan, New York, 1991). 